\title{
Liposomes with conjugates of a calix[4]arene and a Gd-DOTA derivative on the outside surface; an efficient potential contrast agent for MRI $\dagger$
}

\author{
Daniel T. Schühle, ${ }^{a}$ Patrick van Rijn, ${ }^{b}$ Sophie Laurent, ${ }^{c}$ Luce Vander Elst,${ }^{c}$ \\ Robert N. Muller, ${ }^{c}$ Marc C. A. Stuart, ${ }^{d}$ Jürgen Schatz ${ }^{e}$ and Joop A. Peters ${ }^{* a}$
}

Received 24th February 2010, Accepted 20th April 2010

First published as an Advance Article on the web 13th May 2010

DOI: $10.1039 / \mathbf{c 0 c c 0 0 1 0 7 d}$

Paramagnetic liposomes used as contrast agents in magnetic resonance imaging (MRI) often suffer from low efficacies because of slow water diffusion through the membrane. We present an approach to overcome this limitation by incorporation of a calix[4]arene based agent that expresses the chelates towards the bulk water.

Paramagnetic Gd(III) complexes are widely used contrast agents (CAs) in magnetic resonance imaging (MRI). They function by a shortening of the longitudinal relaxation time ( $\left.T_{1, \mathrm{obs}}\right)$ of surrounding water protons, which is determined by the local concentration of the CA ([Gd]) and its relaxivity $\left(r_{1}\right)$ (eqn (1)).

$$
\frac{1}{T_{1, \mathrm{obs}}}=\frac{1}{T_{1, \text { tissue }}}+r_{1}[\mathrm{Gd}]
$$

Whereas $r_{1}$ can be enhanced by, for instance, an increase of the size and therefore of the rotational correlation time $\left(\tau_{R}\right)$ of the CA, a higher [Gd] can be achieved by the introduction of targeting functions to the agent and/or the attachment of several Gd-chelators to a molecular platform leading to a higher Gd-payload per molecule CA. ${ }^{1,2}$ Self-assembled CAs are very promising since they do not only allow the delivery of a huge amount of chelates to the site of interest, they also result in relatively large systems with favorably long $\tau_{\mathrm{R}}{ }^{3}$ Moreover, micelles and liposomes of proper sizes are able to accumulate selectively in tumor tissue by enhanced permeability and retention.

Liposomes with MRI CAs included in their membranes offer the additional possibility to entrap therapeutics or diagnostics for other molecular imaging techniques (e.g. PET

${ }^{a}$ Delft University of Technology, Department of Biotechnology, Biocatalysis and Organic Chemistry, Julianalaan 136,

2628 BL Delft, The Netherlands. E-mail: j.a.peters@tudelft.nl; Tel: +31152785892

${ }^{b}$ Delft University of Technology, Department of Chemical Engineering, Self Assembling Systems, Julianalaan 136, 2628 BL Delft, The Netherlands

${ }^{c}$ Department of General, Organic and Biomedical Chemistry, University of Mons, Place du Parc, 20 B-7000 Mons, Belgium

${ }^{d}$ University of Groningen, Groningen Biomolecular Sciences and Biotechnology Institute, Electron Microscopy Group, Nijenborgh 4, 9747 AG Groningen, The Netherlands

${ }^{e}$ University of Erlangen-Nürnberg, Department of Chemistry and Pharmacy, Henkestraße 42, 91054 Erlangen, Germany

$\dagger$ Electronic supplementary information (ESI) available: Synthesis of 1, preparation of liposomes, characterization of liposomes with DLS, SEC, cryo-TEM and DSC along with the model used for simulations and the results of simulations and fittings. See DOI: 10.1039/c0cc00107d and SPECT) in their cavities. ${ }^{4}$ A disadvantage of liposome systems with MRI CAs incorporated in the membrane is that in the systems reported to date, half of the Gd-chelate molecules are oriented inside. These Gd-chelates have poor relaxivities caused by the low water permeability $\left(P_{\mathrm{w}}\right)$ of the hydrophobic membranes. ${ }^{5}$ This is limiting significantly the overall relaxivity. ${ }^{6}$ Higher $r_{1}$ can be reached by the design of systems with higher $P_{\mathrm{w}}$, but these may have decreased serum stability. Here, we report the preparation and characterization of a system with very high relaxivity by inclusion of calix[4]arene 1 (Fig. 1) into the outer layer of the membrane of DSPC/DSPE-PEG2000-OMe/cholesterol liposomes.

Calix[4]arene 1 was synthesized by alkylation of 5,11,17,23tetra-tert-butyl-25,27-dihydroxy-26,28-dipropoxy-calix[4]-arene with octadecylbromide. Ipsonitration and subsequent reduction yielded a tetra-amino-calix[4]arene that was coupled with tert-butyl-DO3A-monoacid. After deprotection of the tertbutyl functions, the ligand was complexed with Gd(III).

Since the presence of $\mathbf{1}$ during the lipid film preparation leads to disruption of the vesicles, it was impossible to prepare the liposomes by mixing all components prior to the lipid film preparation. Therefore, first a mixture of DSPC/DSPEPEG2000-OMe/cholesterol was dissolved in $\mathrm{CHCl}_{3}$ and the solvents were removed to yield a lipid film. After resuspension in HEPES buffer, $8.8 \mathrm{~mol} \%$ of $\mathbf{1}$ was added and the mixture was extruded at $85{ }^{\circ} \mathrm{C}$ subsequently through filters with diameters of 200 and $100 \mathrm{~nm}$. Dynamic Light Scattering (DLS) showed that the liposomes obtained had a narrow size distribution $(\mathrm{PDI}<0.1)$ and an average hydrodynamic radius of $100 \mathrm{~nm}$ (Fig. S2 in ESI $\dagger$ ).

This was confirmed by transmission electron microscopy (cryo-TEM) and size exclusion chromatography (SEC). The

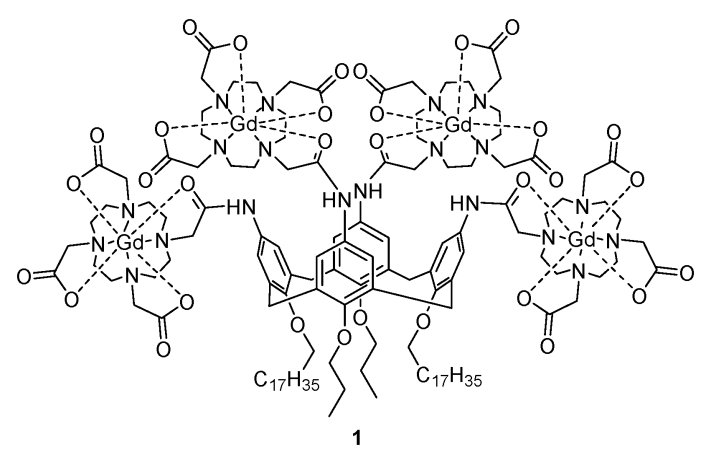

Fig. 1 Amphiphilic calix[4]arene 1. 


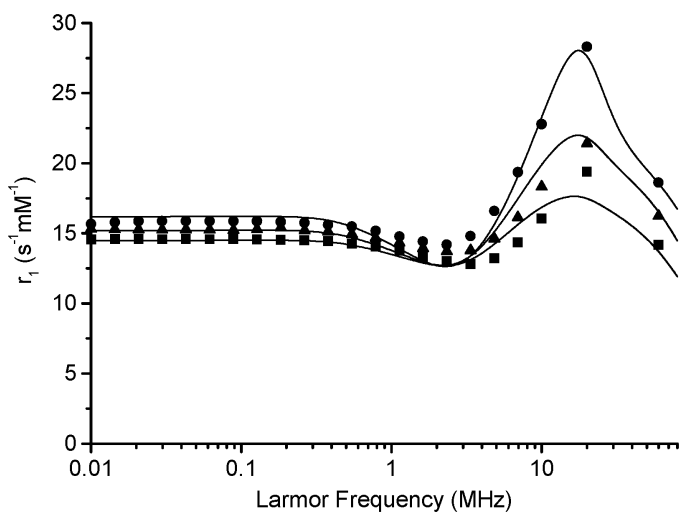

Fig. 2 NMRD profiles of liposomes containing $8.8 \mathrm{~mol} \% \mathbf{1}$ at $25^{\circ} \mathrm{C}$ (squares), $37{ }^{\circ} \mathrm{C}$ (triangles) and $55^{\circ} \mathrm{C}$ (circles).

shift in the phase transition temperature, $T_{\mathrm{m}}$ as determined by differential scanning calorimetry, from $51.6{ }^{\circ} \mathrm{C}$ for a blank sample to $48.4{ }^{\circ} \mathrm{C}$ for the sample containing 1 is an indication that $\mathbf{1}$ is included in the liposomal bilayer. The stability of these liposomes was investigated by monitoring the size and polydispersity of samples stored at $4{ }^{\circ} \mathrm{C}$ in time. Even after 8 weeks, no change was observed suggesting that the particles are highly stable, which may be ascribed to the presence of cholesterol $(33 \mathrm{~mol} \%)$ and to the steric protection by PEGylation.

Nuclear Magnetic Relaxation Dispersion (NMRD) is one of the most important techniques used to characterize MRI CAs. ${ }^{7}$ The field dependence of $r_{1}$ gives important information on the parameters governing the $r_{1}$ of a given system. The NMRD profiles of the presently studied liposomes show local maxima at about $20 \mathrm{MHz}$, which is typical for systems with a long $\tau_{\mathrm{R} \cdot}{ }^{1,2}$ For previously reported liposomal agents having equal amounts of Gd-DTPA-BSA pointing outward and inward from the membrane, NMRD profiles typically show a steep increase of $r_{1}$ with temperature around $T_{\mathrm{m}} \cdot{ }^{8,9}$ This is ascribed to the increase of $P_{\mathrm{w}}$ with temperature leading to an increased contribution to the overall $r_{1}$ of the inward pointing Gd-chelates. Above $T_{\mathrm{m}}, P_{\mathrm{w}}$ is normally high enough to ensure sufficient water exchange between in- and outside the liposomes so that the permeability is no longer limiting the relaxivity. By contrast, liposomes containing $\mathbf{1}$ only show a gradual relatively small increase in $r_{1}$ with temperature, even when $T_{\mathrm{m}}$ of the membranes is exceeded (Fig. 2).

This and the overall very high relaxivities compared to both those of the previously studied monomeric tetrapropyl analogue of $\mathbf{1}^{10}$ and Gd-DTPA-BSA/DSPC/DSPE-PEG2000$\mathrm{OMe} /$ cholesterol suggest that either $P_{\mathrm{w}}$ is extremely high and thus, in the presently studied system, there is no quenching effect due to the membrane and/or that a significant amount of $\mathrm{Gd}$ is oriented towards the bulk water. Therefore, relaxometric $P_{\mathrm{w}}$ measurements were performed on the diamagnetic Y(III) analog of the liposomes according to the procedure described by Terreno. ${ }^{11}$ The $P_{\mathrm{w}}$ of this sample $\left(17.3 \times 10^{-5} \mathrm{~cm} \mathrm{~s}^{-1}\right)$ is too low for efficient water transport. This in combination with the overall high relaxivity suggests that the majority of the Gd-chelates are pointing outward in the presently studied system.
Further support for the predominant location of the Gd-chelates on the outer layer of the membrane was obtained by simulations of the relaxivity at $0.01 \mathrm{MHz}$ as a function of the temperature. These were performed by means of a two-step model that considers the system as an aqueous solution of Gd-chelates in the interior of the liposome with the included water molecules being in exchange with the Gd-chelates and the bulk water outside of it. With the use of parameters estimated from those for the previously studied calix[4]arene systems ${ }^{10}$ and assuming that $50 \%$ of the chelates are located inside the vesicles $\left(f_{\text {in }}=0.5\right)$, a temperature jump in relaxivity of $5 \mathrm{~s}^{-1} \mathrm{mM}^{-1}$ is expected if the residence time of water in the cavity of the liposomes decreases from 9.1 (the value corresponding to $P_{\mathrm{m}}$ ) to $0.1 \mathrm{~ms}$. By contrast, the relaxivity remains almost constant when $f_{\text {in }}$ is zero (see ESI $\dagger$ ). From these simulations, it may be concluded that at least $80 \%$ of the Gd-chelates are oriented towards the outside of the liposomes. The slight increase of $r_{1}$ with temperature is a consequence of an accelerated exchange rate of water directly bound to the metal center $\left(k_{\mathrm{ex}}\right)$. Finally, a good simultaneous fit of the NMRD profiles was obtained with the SolomonBloembergen-Morgan theory adapted with the Lipari-Szabo approach to take into account the effects of local mobility assuming that all Gd-chelates are located in the outer membrane layer (see ESI $\dagger$ ). The best-fit parameters (see Fig. 2 (lines) and $\mathrm{ESI} \dagger$ ) are in the range to be expected based on values obtained for the previously studied low-molecular weight calix[4]arene system. ${ }^{10}$

Attempts to prepare liposomes with about equal amounts of chelate on the inner and outer membrane layer with the use of the "classical" preparation protocol ${ }^{6,12}$ failed; cryo-TEM images showed that complex mixtures of aggregates with a high content of disk-like micelles (bicelles) along with vesicles that were not completely closed or multilamellar (see ESI $\dagger$ ) were always obtained.

In conclusion, we present a novel approach towards stable liposomal MRI contrast agents with high relaxivity. The calix[4]arene derivative is mainly incorporated at the outer surface of the vesicles. The Gd(III) ion is chelated with DOTA, which guarantees a high kinetic stability, which is an advantage in view of the toxicity of free Gd(III). Apparently, the steric demands of $\mathbf{1}$ favor its inclusion in the outer layer of the membrane. The bulkiness of $\mathbf{1}$ practically excludes a flip-flop of the Gd-chelates from the outer to the inner layer of the membrane. ${ }^{13}$ The cavity of these liposomes is available for therapeutics and other diagnostics and, therefore, these systems may have potential as dual modality probes for molecular imaging and as theranostics.

Thanks are due to K. Djanashvili, U. Hanefeld, D. S. Abou, H. T. Wolterbeek (Delft University of Technology) for helpful discussions, to F. Mayer for chromatographic analyses, and to C. Bonnet (CNRS, Orléans, France) for performing some relaxometric measurements. This work was financially supported by the European Union through a Marie-Curie training site host fellowship (MEST-CT-2004-7442), COST action D38 (Metal-Based Systems for Molecular Imaging Applications), and the European Network of Excellence "European Molecular Imaging Laboratories" (LSCH-2004503569). 


\section{Notes and references}

1 É. Tóth and A. E. Merbach, The Chemistry of Contrast Agents in Medical Magnetic Resonance Imaging, Wiley, Chichester, 2001.

2 P. Caravan, J. J. Ellison, T. J. McMurry and R. B. Lauffer, Chem. Rev., 1999, 99, 2293.

3 A. Accardo, D. Tesauro, L. Aloj, C. Pedone and G. Morelli, Coord. Chem. Rev., 2009, 253, 2193.

4 W. T. Phillips, B. A. Goins and A. Bao, Wiley Interdiscip. Rev. Nanomed. Nanobiotechnol., 2009, 1, 69.

5 W. J. M. Mulder, G. J. Strijkers, G. A. F. van Tilborg, A. W. Griffioen and K. Nicolay, NMR Biomed., 2006, 19, 142.

6 S. Laurent, L. vander Elst, C. Thirifays and R. N. Muller, Eur. Biophys. J., 2008, 37, 1007.

7 D. H. Powell, O. M. Ni Dhubhghaill, D. Pubanz, L. Helm, Y. S. Lebedev, W. Schlaepfer and A. E. Merbach, J. Am. Chem. Soc., 1996, 118, 9333.
8 G. J. Strijkers, W. J. M. Mulder, R. B. van Heeswijk, P. M. Frederik, P. Bomans, P. C. M. M. Magusin and K. Nicolay, Mag. Reson. Mater. Phys. Biol. Med., 2005, 18, 186.

9 S. Hak, H. M. H. F. Sanders, P. Agrawal, S. Langereis, H. Grüll, K. M. Keizer, F. Arena, E. Terreno, G. J. Strijkers and K. Nicolay, Eur. J. Pharm. Biopharm., 2009, 72, 397.

10 D. T. Schühle, J. Schatz, S. Laurent, L. Vander Elst, R. N. Muller, M. C. A. Stuart and J. A. Peters, Chem.-Eur. J., 2009, 15, 3290.

11 E. Terreno, A. Sanino, C. Carrera, D. Delli Castelli, G. B. Giovenzana, A. Lombardi, R. Mazzon, L. Milone, M. Visigalli and S. Aime, J. Inorg. Biochem., 2008, 102, 1112.

12 S. Laurent, L. Vander Elst, C. Thirifays and R. N. Muller, Langmuir, 2008, 24, 4347.

13 M. Nakano, M. Fukado, T. Kudo, N. Matsuzaki, T. Azuma, K. Sekine, H. Endo and T. Handa, J. Phys. Chem. B, 2009, 113, 6745 . 